\title{
Undergraduate Advanced Laboratory Studies on Supersonic Nozzle Flow
}

\author{
Keith R. Stein*, Connor D. Fredrick* and Richard W. Peterson* \\ *Department of Physics, Bethel University, 3900 Bethel Drive, St. Paul, MN 55112
}

\begin{abstract}
Undergraduate studies are carried out in the advanced laboratory to examine the supersonic flow from an axisymmetric converging-diverging nozzle. Flow is initiated by the rupture of a diaphragm and exits from a small nozzle (with a $0.95 \mathrm{~cm}$ exit diameter) into standard atmospheric conditions from a one gallon (3.875 liter) tank. Compressible flow simulations are carried out for the nozzle and comparisons are made to experiments based on high-speed video shadowgraph imaging and dual-beam interferometry. The multifaceted approach based on simulation and experiments exploits the distinct strengths of the different methods to provide a more comprehensive description of the nozzle flow than is achievable by the individual approaches.
\end{abstract}

Keywords: shadowgraph imaging, high-speed video, heterodyne interferometry, computational fluid dynamics, advanced undergraduate laboratory.

PACS: $47.40 \mathrm{Ki}, 47.80 . \mathrm{Jk}, 07.60 . \mathrm{Ly}$

\section{INTRODUCTION}

Advanced undergraduate laboratories that strive to effectively merge ongoing research and academic studies into a productive and engaging experience are faced with a number of fundamental challenges. This study of compressible nozzle flow dynamics is based on an integration of computational physics, high-speed video (HSV) imaging and analysis, and quantitative gas density measurements from time-resolved interferometry ${ }^{1}$. This integrated approach has the flexibility to encourage student team creativity while enhancing project-based laboratories associated with several undergraduate courses, including fluid dynamics, computational methods, and physical optics.

Several aspects of this experiment are pedagogically appealing for undergraduates in physics and engineering. While the phenomenon studied is relatively straightforward (and very simple) to initiate, students quickly come to appreciate the underlying complexity of the highly transient supersonic flow behavior. HSV shadowgraphs near the nozzle (at up to 100,000 frames/sec) serve to stimulate student interest and intuition regarding the qualitative flow behavior in space and time before computations and predictions are made to predict the expected result. Depending on the academic focus of the class using the system, such qualitative imaging provides an ideal background for either computational physics (compressible flow simulation) or time-resolved fluid density measurements from heterodyne interferometry. The complementary nature of these approaches is often best appreciated through the diverse physics and engineering backgrounds within the student teams.
Teams quickly learn that some of their members will thrive on the computational challenges, while others will exhibit the experimental perseverance that enables the optical diagnostics.

\section{EXPERIMENTAL APPARATUS}

The nozzle flow apparatus was designed and constructed as part of an advanced laboratory project in the Bethel University junior-level fluid mechanics course. This course has a lab component that comes in the form of two open-ended team projects; a minor project that is often exploratory in nature and a major project that strives to provide the student with a research-like experience. Major projects frequently build off of past projects or ongoing research activities. Additionally, projects are often enhanced by the experiences and skills that project team members bring from other advanced laboratory courses or from student research. For example, the experiments described in this paper relied on student skills obtained through advanced laboratory project work in the Bethel University optics course.

The apparatus is constructed with a 1-gallon (3.875 liter) air pressure tank and a converging-diverging nozzle (see Fig. 1). A Mylar diaphragm is secured in a rupture disk holder that mates the tank to the nozzle and ruptures at tank gage pressures of approximately $4.48 \times 10^{5} \mathrm{~Pa}$, initiating the flow through the nozzle. The nozzle is designed with a throat to exit area ratio of 0.71 , resulting in a Mach 1.8 exit flow condition.

Following the rupture of the diaphragm, the compressible flow dynamics downstream from the nozzle are categorized in three stages: a highly 
transient start-up flow, quasi-steady supersonic nozzle flow, and the nozzle blow-down. This experiment focuses on the transient flow from the nozzle during the initial stage, including the initial shock wave and 200 microseconds of supersonic flow following the shock.

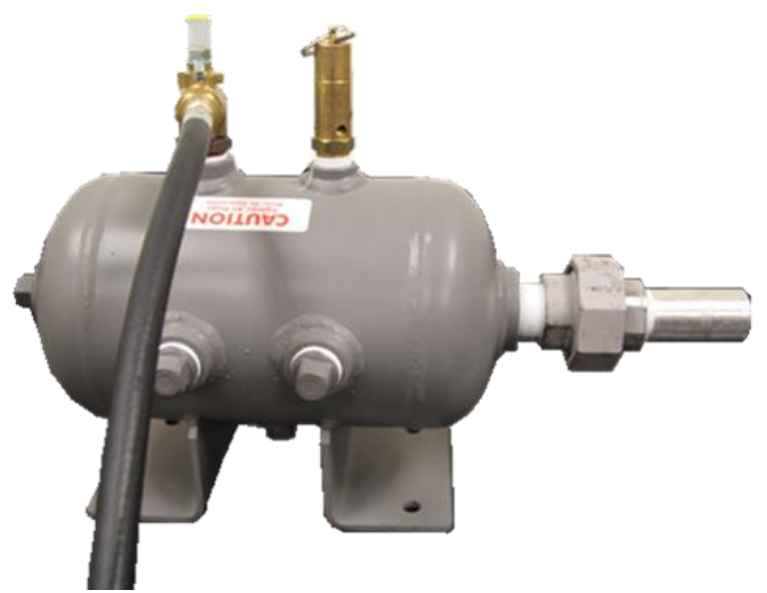

FIGURE 1. Nozzle flow apparatus: Pressure tank and nozzle. The 1-gallon ASME-code horizontal compressed air tank is available through Mcmaster.com.

\section{ADVANCED LABORATORY EXPERIMENTS AND SIMULATIONS}

In this section we describe a threefold approach for characterization of the startup flow from the nozzle based on finite element compressible flow simulations, HSV shadowgraphy, and phase-detecting interferometry.

\section{Compressible Flow Simulation}

Nozzle flow simulations are carried out for unsteady, axisymmetric compressible flow using COMSOL $\AA$. The flow is driven by an initial pressure condition that is representative of the experimental condition upon rupture of the diaphragm, with the location of the diaphragm serving as the boundary between the high and low pressure regions. No-slip, thermally insulated boundary conditions are prescribed at all boundaries, except for the symmetry axis. The axisymmetric model (rotated for clarity) and the initial conditions are depicted in Fig. 2.

The fluid domain is discretized with an unstructured triangular mesh of approximately 200,000 elements. COMSOL finite element computations provide solutions at mesh nodes for the timedependent, compressible flow equations describing conservation of mass, momentum, and energy for an

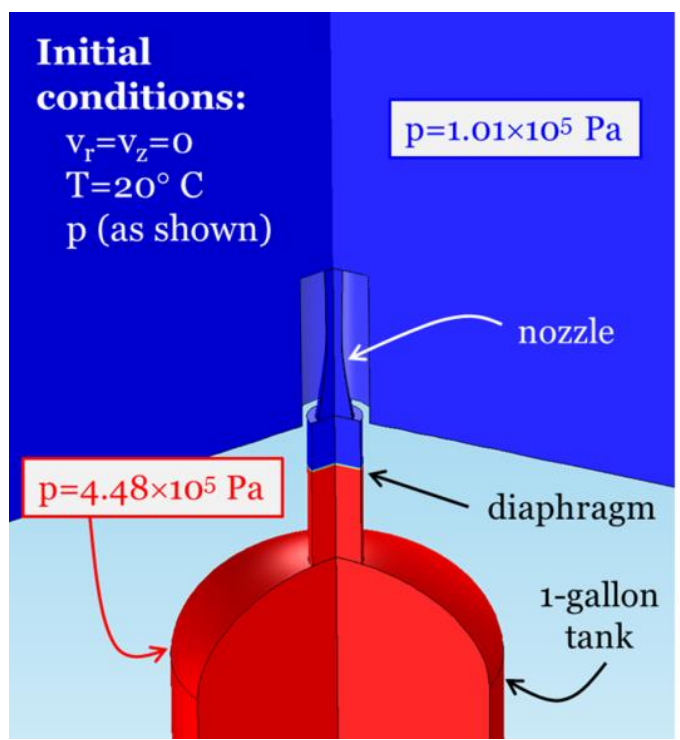

FIGURE 2. Compressible flow simulation: Axisymmetric model and initial conditions.

ideal gas. The gradient of the computed density field is displayed in Fig. 3 at times of $50 \mu$ s and $90 \mu$ s after the initial shock exits the nozzle. The density fields, mirrored about the symmetry axis for clarity, portray the initial shock wave and development of the supersonic flow from the nozzle.

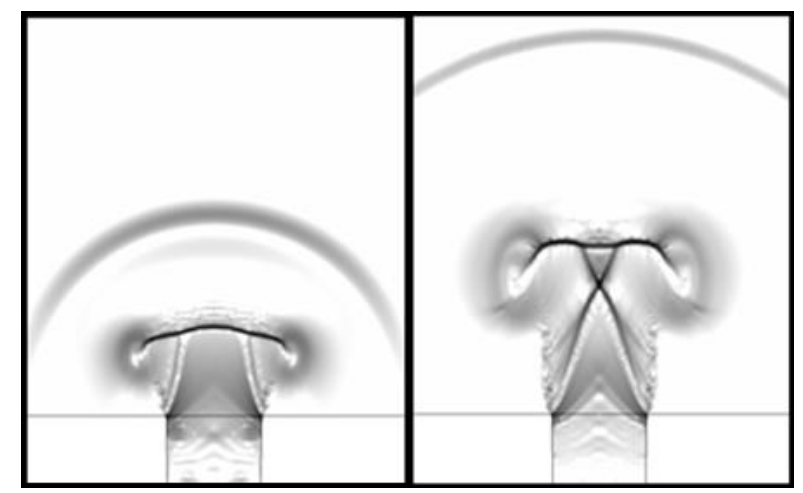

FIGURE 3. Density gradient field $50 \mu$ s and $90 \mu$ s after the initial shock wave exit the nozzle.

\section{High-Speed Video Shadowgraphy}

HSV shadowgraph imaging ${ }^{2,3}$ is utilized to study the initial flow from the nozzle. Light from a $400 \mathrm{~mW}$ $532 \mathrm{~nm}$ laser passes through an objective lens and spatial filter as shown in Fig. 4. The diverging beam passes through the nozzle exit flow region and is projected on a frosted glass window for imaging. 


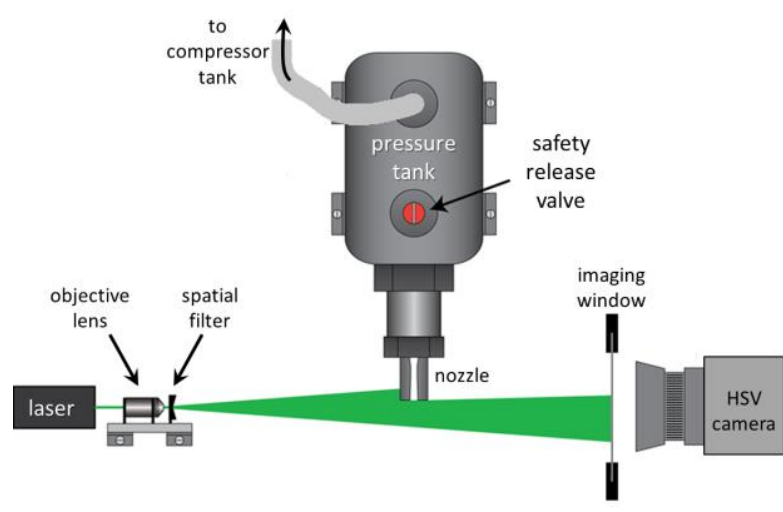

FIGURE 4. Shadowgraph imaging setup.

The shadowgraph projection is recorded at 100,000 frames/second with a $0.4 \mu$ s shutter using a NAC Memrecam HX-3 digital high-speed video camera. Frame resolutions of approximately 200x200 pixels are achieved at these settings. The shadowgraph video is useful in characterizing the evolution of the nozzle jet flow. Images from the shadowgraph sequence are shown in Fig. 5 at times of $50 \mu$ s and $90 \mu$ s after the initial shock exits the nozzle, for comparison with the computed density fields shown in Fig. 3.

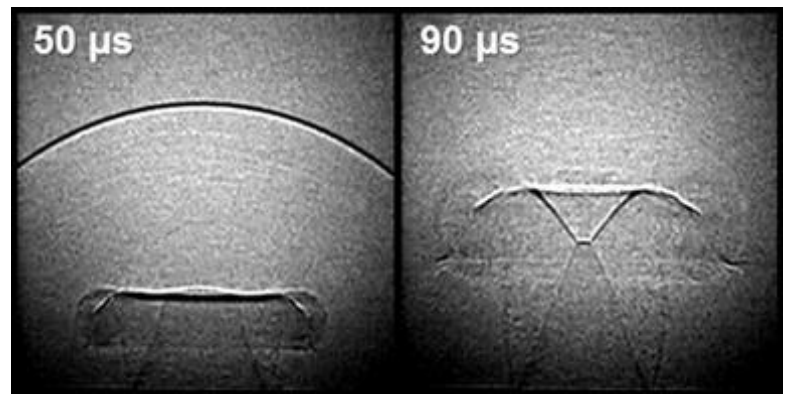

FIGURE 5. Shadowgraph images of the initial shockwave and nozzle flow corresponding to the simulation times shown in Fig. 3. The images were extracted from individual frames from a 100,000 frame/sec video using a NAC Memrecam HX-3 camera.

\section{Phase-Detecting Interferometry}

Although the high-speed video describes the evolution of the nozzle flow, imaging with sufficient pixel resolution is limited to around 100,000 frames per second. Phase-detecting interferometry offers an experimental method for quantitative analysis of the highly-transient nozzle startup flow at millions of samples per second.

A LabVIEW ${ }^{\circledR}$ enhanced system, utilizing a heterodyne interferometer (see Fig. 6), monitors the rapidly changing optical path length along a selected probe-line path in the nozzle vicinity. An acousto-optic modulator (model AOM-40, IntraAction Corp.) frequency-shifts a stabilized He-Ne laser at $633 \mathrm{~nm}$ by $40 \mathrm{MHz}$. This light passes into the arms of the interferometer and is retroreflected back through the AOM. When the frequency shifted light is recombined with original incident laser light, any phase changes between the interferometer arms are encoded in $80 \mathrm{MHz}$ beat signals on twin DC-125 MHz, low noise photodetectors (New Focus model 1811, Newport Corp.). Highly dynamic variations in air density from the initial shock wave and compressible flow dynamics produce changes in the refractive index, which are observed as changes to the optical path length or as changes to the phase of the laser light. Such a heterodyne system has diverse applications, including surface metrology ${ }^{4}$ and fluid shock wave ${ }^{1,5}$ diagnostics. An RF mixer and LABVIEW VI decode and extract these phase shifts as a function of time.

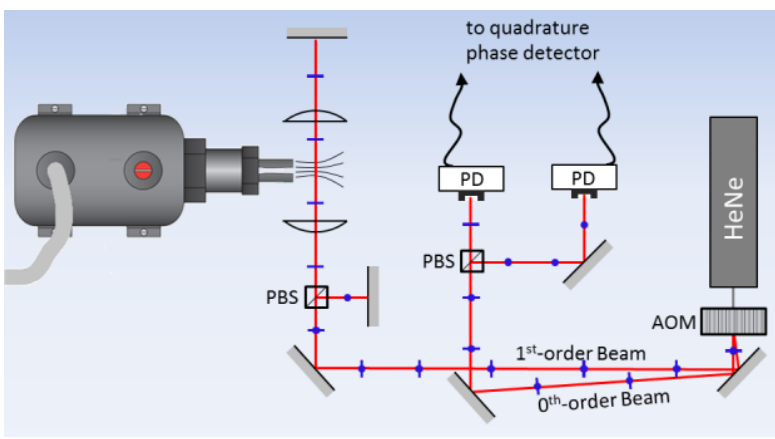

FIGURE 6. Heterodyne interferometer setup.

Interferometer measurements of total phase shift detected at various distances $(10 \mathrm{~mm}, 15 \mathrm{~mm}$, and $20 \mathrm{~mm}$ ) from the nozzle exit are shown Fig. 7. The initial peak on the graph corresponds to the initial shock wave, and is followed by a rapid drop in phase, signifying the low pressure flow region following the shock. The dashed lines correspond to predicted phase shifts from the flow simulation and are evaluated by integration through the computed density field. The comparison shows a strong correlation in the temporal spacing of the shock waves and of the phase shift due to the low pressure flow following the initial shock. However, the comparison shows a noticeable mismatch in the phase shift produced by the incident shock wave. This is a result of modeling the rupture of the diaphragm in the computer simulation as an instantaneous event, which is more abrupt than the physical rupture event. 


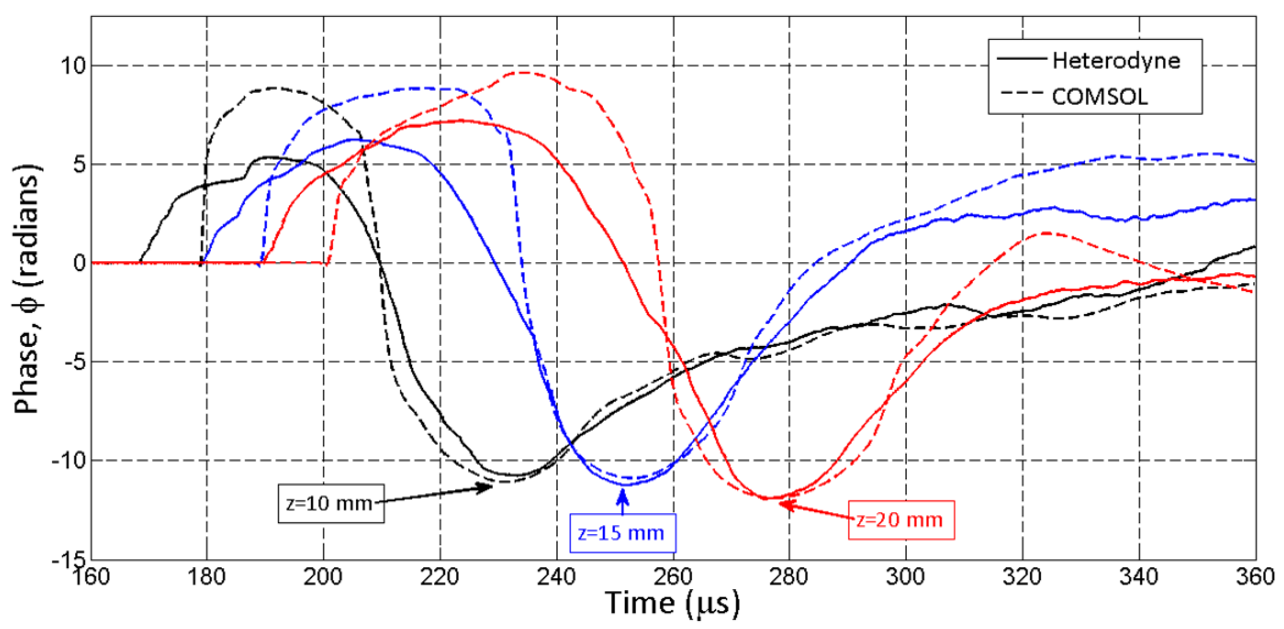

FIGURE 7. Comparison between total phase shift from the heterodyne interferometer and the compressible flow simulation.

\section{CONCLUSION}

An integrated approach to study compressible nozzle flow dynamics utilizes computational physics, HSV imaging and analysis, and quantitative gas density measurements from time-resolved interferometry. This strategy is pedagogically attractiveinterferometry. This strategy is pedagogically attractive for undergraduate students in physics and engineering, while addressing some of the fundamental challenges for advanced undergraduate laboratories striving to provide an engaging lab experience that unites ongoing research and academic studies. The integrative approach is wellsuited and appreciated in project teams composed of students with diverse physics and engineering backgrounds. Project teams quickly recognize that some of the members will thrive on the computational challenges, whereas others exhibit experimental skills and perseverance that is well-suited for optical diagnostics. This complementary approach results in a richer and fuller analysis of the nozzle flow than is achievable by the individual approaches.

\section{ACKNOWLEDGEMENTS}

This work was supported in part by the National Science Foundation under Grant No. 1245573 and by the Minnesota Space Grant Consortium in accordance with NASA's emphasis on giving undergraduates hands-on experiences in research, especially in areas of research applicable to aeronautics and space science. We are grateful to Kent Peterson and NAC Image Technology for their support with the NAC Memrecam HX-3 camera.

\section{REFERENCES}

1. C.D. Fredrick et al., in Computational Methods and Experimental Measurements XVII, edited by G.M. Carlomagno, D. Poljak, and C.A. Brebbia (WIT Press, 2015).

2. G.S. Settles, Schlieren and Shadowgraph Techniques: Visualizing Phenomena in Transparent Media. SpringVerlag: Berlin and New York, 2001.

3. H.W. Liepmann and A. Roshko, Elements of Gasdynamics. Dover: New York, 1993.

4. T. Carlson et. al, Appl. Optics, 36 (28), 7162-7171 (1997).

5. G. Olson et. al, Am. J. of Phys., 74 (12), 1071-1076 (2006). 